\title{
CONCORDANCIA DIAGNÓSTICA ENTRE SIETE DEFINICIONES DE SÍNDROME METABÓLICO EN ADULTOS CON SOBREPESO Y OBESIDAD
}

\author{
Eduardo Cabrera-Rode ${ }^{1, a}$, Beatriz Stusser ${ }^{1, b}$, Wenny Cálix ${ }^{1, b}$, Neraldo Orlandi ${ }^{1, b}$, Janet Rodríguez ${ }^{1, c}$, \\ Ileana Cubas-Dueñas ${ }^{1, \mathrm{~d}}$, Ragmila Echevarría ${ }^{1, \mathrm{c}}$, Aimee Álvarez $^{1, \mathrm{e}}$
}

\begin{abstract}
RESUMEN
Objetivos. El objetivo del presente estudio fue determinar el grado de concordancia diagnóstica entre siete definiciones de síndrome metabólico (SM) en un grupo de adultos con sobrepeso y obesos. Material y Métodos. Se estudiaron 350 sujetos con edades comprendidas entre 19 y 70 años que fueron reclutados consecutivamente de una consulta para sujetos con sobrepeso y obesidad. Se emplearon las definiciones de SM según OMS (Organización Mundial de la Salud), EGIR (Grupo Europeo para el estudio de la resistencia a la insulina), NCEP-ATPIII (Panel de Tratamiento de Adultos), AHA/NHLBI (Asociación Americana del Corazón), IDF (Federación Internacional de Diabetes), JIS (Declaración provisional conjunta), así como los criterios de Szabo. La concordancia entre las definiciones fue calculada con el coeficiente Kappa. La resistencia a la insulina (RI) fue evaluada mediante el índice HOMA. Resultados. Según los criterios de Szabo, OMS, EGIR, NCEP-ATPIII, AHA/NHLBI, IDF y la JIS, la frecuencia de SM fue del 74,3\%, 42,0\%, $46,8 \%, 56,0 \%, 52,9 \%, 58,6 \%$ y $58,6 \%$, respectivamente. La concordancia entre los criterios de Szabo y la AHA/NHLBI fue de 0,559 , mientras que el coeficiente kappa entre los criterios de Szabo, y el resto de las guías (NCEP-ATPIII, IDF, JIS) fue de 0,612 a 0,657, respectivamente. La concordancia de la OMS con las demás guías fue entre 0,358 y 0,422, pero con la EGIR la concordancia fue de 0,602. La RI se distribuyó de manera similar en todas las guías. Conclusiones. Existe una considerable concordancia entre las guías NCEP-ATPIII, IDF, JIS y el SM según criterios de Szabo. EI SM según criterios de Szabo pudiera ser otra alternativa para la pesquisa activa del SM en poblaciones.
\end{abstract}

Palabras claves: Síndrome X metabólico; Prevalencia; Sobrepeso; Obesidad; Resistencia a la insulina (fuente: DeCS BIREME).

\section{DIAGNOSTIC CONCORDANCE BETWEEN SEVEN DEFINITIONS OF METABOLIC SYNDROME IN OVERWEIGHT AND OBESE ADULTS}

\begin{abstract}
Objectives. The aim of this study was to determine the level of diagnostic concordance between seven definitions of metabolic syndrome (MS) in a group of overweight and obese adults. Materials and Methods. 350 subjects aged from 19 to 70 years were recruited for study from a clinic for overweight and obese subjects. The definitions of MS used were those given by the WHO (World Health Organization), EGIR (European Group for the Study of Insulin Resistance), NCEPATPIII (Adult Treatment Panel), AHA/NHLBI (American Heart Association), IDF (International Diabetes Federation), and JIS (Joint Interim Statement) as well as the Szabo criteria. Concordance between the definitions was calculated with the Kappa coefficient. Insulin resistance (IR) was assessed using the HOMA-IR index. Results. According to the Szabo, WHO, EGIR, NCEP-ATPIII, AHA/NHLBI, IDF, and JIS criteria, MS frequency was $74.3 \%, 42.0 \%, 46.8 \%, 56.0 \%, 52.9 \%$, $58.6 \%$, and $58.6 \%$, respectively. The concordance between the Szabo and AHA/NHLBI criteria was 0.559 , while the Kappa coefficient between the Szabo criteria and the rest of the guides (NCEP-ATPIII, IDF, and JIS) was from 0.612 to 0.657 , respectively. The concordance of the WHO with the EGIR was 0.602 , but it was between 0.358 and 0.422 with the other guidelines. IR was distributed similarly in all guidelines. Conclusions. There is a considerable concordance between the NCEP-ATPIII, IDF, and JIS guidelines and the Szabo criteria. The Szabo criteria could be an option for the active surveillance of MS in populations.
\end{abstract}

Key words: Metabolic X syndrome; Prevalence; Overweight; Obesity; Insulin resistance (source: MeSH NLM).

\footnotetext{
Instituto Nacional de Endocrinología. La Habana, Cuba.

Doctor en Ciencias, ${ }^{\mathrm{b}}$ médico endocrinólogo, ${ }^{\mathrm{c}}$ licenciada en Ciencias Tecnológicas de la Salud, ${ }^{\mathrm{d}}$ médico inmunólogo, ${ }^{\mathrm{e}}$ máster en Ciencias Bioquímicas. Recibido: 01/09/2016 Aprobado: 22/02/2017 En línea: 23/03/2017
}

Citar como: Cabrera-Rode E, Stusser B, Cálix W, Orlandi N, Rodriguez J, Cuba-Dueñaz I, et al. Concordancia diagnóstica entre siete definiciones de síndrome metabólico en adultos con sobrepeso y obesidad. Rev Peru Med Exp Salud Publica. 2017;34(1):19-27. doi: 10.17843/rpmesp.2017.341.2763 


\section{INTRODUCCIÓN}

El término de síndrome metabólico (SM) nace de la combinación de una serie de factores (obesidad abdominal, anomalías en el metabolismo de la glucosa, hiperinsulinemia, hipertensión y dislipidemia) que, agrupados, incrementan el riesgo de desarrollar diabetes tipo 2 (DM2), enfermedad coronaria y enfermedad cerebrovascular (ECV), entre otras ${ }^{(1-3)}$.

En 2004, Reaven (4) postuló que la resistencia a la insulina (RI) desempeñaba un papel primordial en fisiopatología de la enfermedad coronaria; de ahí que también comenzara a denominársele como síndrome de resistencia a la insulina (SRI), aunque actualmente se prefiere utilizar el término de SM para referirse a él ${ }^{(1-4)}$.

Diversas han sido las propuestas para diagnosticar al SM ${ }^{(2,3)}$. Desde 1998 muchas organizaciones han publicado criterios diagnósticos para adultos, incluyendo o no la Rl, en un intento por aclarar y estandarizar la definición del SM ${ }^{(5-10)}$. Estas definiciones coincidieron en reconocer a la obesidad abdominal como indicador de RI, con diferencias en los puntos de corte utilizados ${ }^{(5-10)}$. En el 2008 se propuso los criterios de SM según Szabo ${ }^{(11)}$.

La prevalencia del SM ha aumentado drásticamente en los últimos años por el aumento de la obesidad y los cambios en los hábitos dietéticos de la población. EI SM es probablemente el resultado de la influencia de los factores genéticos y medioambientales, así como los hábitos dietéticos y la insuficiente actividad física ${ }^{(2,3,12-14)}$.

En Estados Unidos entre 1994 y el 2010 la prevalencia del SM en adultos se incrementó de 23 al $34 \%$, periodo en que también se incrementaron la obesidad y la inactividad física ${ }^{(3,13)}$. En varias ciudades de Centroamérica, la prevalencia del SM de acuerdo a los criterios de la National Cholesterol Education Program Adult Treatment Panel III (NCEP-ATPIII) osciló entre el 23 al $35,1 \%{ }^{(14)}$.

En Cuba se han realizado varios estudios sobre la frecuencia de SM utilizando los criterios de las definiciones de la Asociación Latinoamericana de Diabetes (ALAD) y de la NCEP-ATPIII ${ }^{(15,16)}$. La frecuencia de SM en estas investigaciones fue del $39 \%$ en población general y del $69,5 \%$ en sujetos obesos ${ }^{(15,16)}$.

Numerosos estudios han puesto en evidencia la relación entre obesidad, RI y los diferentes componentes del SM ${ }^{(1-4,17,18)}$. Por lo tanto, los hallazgos sugieren que tanto la obesidad inicialmente, como la RI posteriormente, contribuyen de forma primordial a su desarrollo; sin embargo, en los sujetos obesos la RI puede ser más importante que el grado de obesidad ${ }^{(2-4,19)}$.

La discrepancia en el criterio de obesidad abdominal entre las distintas guías ha conllevado a variaciones en las prevalencias de SM al aplicar cada definición a una misma población (1-3,18,20,21). Sin embargo, las conclusiones han discrepado en los estudios de concordancia donde se analizan estas variaciones entre los sondeos de SM obtenidas con las guías de la Organización Mundial de la Salud (OMS), NCEP-ATPIII, American Heart Association / National Heart, Lung and Blood Institute (AHA/NHLBI), International Diabetes Federation (IDF), y Joint Interim Statement (JIS) ${ }^{(20,21)}$.

Considerando que actualmente en Cuba no se han realizado estudios donde se analice la prevalencia del SM utilizando diferentes definiciones, motivados por la epidemia de obesidad que ha emergido en las últimas décadas ${ }^{(1-3,12,13,20,21)}$ y su relación con la RI, nos proponemos estimar el grado de concordancia diagnóstica entre siete criterios de SM en un grupo de adultos con sobrepeso y obesidad.

\section{MATERIALES Y MÉTODOS}

\section{DISEÑO DEL ESTUDIO}

Estudio observacional y transversal que incluyó a sujetos no diabéticos con sobrepeso (índice de masa corporal [IMC] entre 25,0 y $29,9 \mathrm{~kg} / \mathrm{m}^{2}$ ) y obesos $\left(\mathrm{IMC} \geq 30,0 \mathrm{~kg} / \mathrm{m}^{2}\right)$ según los criterios de la OMS, con edades comprendidas entre los 19 y 70 años, que fueron reclutados consecutivamente de una consulta para sujetos con sobrepeso y obesidad en el período comprendido entre 2014 y el 2015, en la Habana, Cuba. Se excluyeron a las personas con diabetes tipo 1 y 2 , otros tipos específicos de diabetes, además de individuos con enfermedades crónicas asociadas que requirieron tratamiento esteroideo o inmunosupresor.

\section{MUESTRA}

La selección de la muestra se realizó entre personas que aceptaron participar en el estudio realizado en campañas de salud de despistaje de factores de riesgo metabólico en la ciudad de la Habana. Se calculó el tamaño muestral mediante el programa Epidat 3.1. Con una población de 4500 , un nivel de confianza al 95\%, una proporción esperada de obesos de $31,6 \%$, una precisión de muestreo de 0,9 y un efecto de diseño de 0,1 ; se estimó un mínimo de 313 sujetos a incluir en el estudio.

\section{DEFINICIONES DE SÍNDROME METABÓLICO}

Para el diagnóstico de SM se emplearon las definiciones de la OMS ${ }^{(2,5)}$, NCEP-ATPIII ${ }^{(2,6)}$, European Group for the 
Study of Insulin Resistance (EGIR) ${ }^{(2,7)}$, AHA/NHLBI ${ }^{(2,8)}$, IDF ${ }^{(2,9)}$, y la JIS; esta última es una declaración provisional conjunta de varias asociaciones (IDF, AHA, Federación Mundial del Corazón, Sociedad Internacional de Ateroesclerosis, Asociación Internacional para el Estudio de la Obesidad y la NHLBI ${ }^{(2,10)}$. Se detallan las distintas definiciones para SM:

OMS. Presencia de DM2, glucosa en ayunas alterada, tolerancia a la glucosa alterada o RI y al menos dos de los siguientes criterios: índice cintura/cadera (ICC) $>0,9$ en hombres $\mathrm{y}>0,85$ en mujeres o $\mathrm{IMC} \geq 30 \mathrm{Kg} / \mathrm{m}^{2}$; triglicéridos $\geq 1,7 \mathrm{mmol} / \mathrm{L}$; HDL-C $<0,9 \mathrm{mmol} / \mathrm{L}$ en hombres $\mathrm{y}<1,0$ $\mathrm{mmol} / \mathrm{L}$ en mujeres; presión arterial $(P A) \geq 140 / 90 \mathrm{mmHg}$; glucosa en ayunas $\geq 6,1 \mathrm{mmol} / \mathrm{L}^{(2,5)}$.

EGIR. Presencia de RI o valores de insulina en ayunas mayores al percentil 75 en pacientes no diabéticos, más dos de los siguientes criterios: circunferencia de cintura $\geq 94 \mathrm{~cm}$ hombres $\mathrm{y} \geq 80 \mathrm{~cm}$ mujeres; triglicéridos $>2,0 \mathrm{mmol} / \mathrm{L} ; \mathrm{HDL}-\mathrm{C}<1,0 \mathrm{mmol} / \mathrm{L}$ en ambos sexos; $\mathrm{PA} \geq 140 / 90 \mathrm{mmHg}$ o con tratamiento antihipertensivo; glucosa en ayunas $\geq 6,1 \mathrm{mmol} / \mathrm{L}^{(2,7)}$.

NCEP-ATPIII. Presencia de al menos tres de los siguientes factores: circunferencia de cintura $>102 \mathrm{~cm}$ en hombres y $>88 \mathrm{~cm}$ en mujeres; triglicéridos $\geq 1,7 \mathrm{mmol} / \mathrm{L}$ o con tratamiento; HDL-C $<1,0 \mathrm{mmol} / \mathrm{L}$ en hombres $y$ $<1,3 \mathrm{mmol} / \mathrm{L}$ en mujeres o con tratamiento; $\mathrm{PA} \geq 130 / 85$ $\mathrm{mmHg}$; glucosa en ayunas $\geq 6,1 \mathrm{mmol} / \mathrm{L}^{(2,6)}$.

AHA/NHLBI. Presencia de al menos tres de los siguientes factores: circunferencia de la cintura $>102 \mathrm{~cm}$ en hombres $y>88 \mathrm{~cm}$ en mujeres; triglicéridos $\geq 1,7 \mathrm{mmol} / \mathrm{L} \circ \mathrm{con}$ tratamiento; $\mathrm{HDL}-\mathrm{C}<1,0 \mathrm{mmol} / \mathrm{L}$ en hombres $\mathrm{y}<1,3 \mathrm{mmo} / \mathrm{L}$ en mujeres o con tratamiento; PA $\geq 130 / 85 \mathrm{mmHg}$ o diagnóstico previo de HTA; glucosa en ayunas $\geq 5,6 \mathrm{mmol} / \mathrm{L}$ o con tratamiento $(2,8)$.

IDF. Presencia de obesidad abdominal (circunferencia de cintura $\geq 94 \mathrm{~cm}$ hombres $\mathrm{y} \geq 80 \mathrm{~cm}$ mujeres) o IMC $>30 \mathrm{~kg} / \mathrm{m}^{2}$; y al menos dos de los siguientes criterios: triglicéridos $\geq 1,7 \mathrm{mmo} /$ Lo con tratamiento; $\mathrm{HDL}-\mathrm{C}<1,0 \mathrm{mmo} / \mathrm{L}$ en hombres y $<1,3 \mathrm{mmol} / \mathrm{L}$ en mujeres o con tratamiento; PA $\geq 130 / 85 \mathrm{mmHg}$ o con tratamiento; glucosa en ayunas $\geq 5,6 \mathrm{mmol} / \mathrm{L}^{(2,9)}$.

JIS. Presencia de al menos tres de los siguientes factores: obesidad abdominal (circunferencia de la cintura en hombre $\geq 94 \mathrm{~cm}$ y en mujeres $\geq 80 \mathrm{~cm}$ ); triglicéridos $\geq 1,7 \mathrm{mmol} / \mathrm{L} \mathrm{o}$ tratamiento; HDL colesterol $<1,0 \mathrm{mmol} / \mathrm{L}$ en hombres y $<1,3 \mathrm{mmol} / \mathrm{L}$ en mujeres o tratamiento; presión arterial $\geq 130 / 85 \mathrm{mmHg}$ o tratamiento; glucosa en ayunas $\geq 5,6 \mathrm{mmol} / \mathrm{L}$ o tratamiento. ${ }^{(2,10)}$ Además, se aplicó los criterios de SM según Szabo (Tabla 1) ${ }^{(11)}$.
Tabla 1. Criterios para el diagnóstico de síndrome metabólico según Szabo ${ }^{(11)}$

\begin{tabular}{|c|c|c|c|c|c|c|}
\hline \multirow{3}{*}{ Componentes } & \multicolumn{6}{|c|}{ Puntuación } \\
\hline & \multicolumn{2}{|c|}{0} & \multicolumn{2}{|c|}{1} & \multicolumn{2}{|c|}{2} \\
\hline & $\mathrm{H}$ & $M$ & $\mathrm{H}$ & $\mathbf{M}$ & $\mathrm{H}$ & $M$ \\
\hline $\mathrm{PC}(\mathrm{cm})$ & $<94$ & $<80$ & $\geq 94$ & $\geq 80$ & $\geq 102$ & $\geq 88$ \\
\hline $\mathrm{HDL}-\mathrm{C}(\mathrm{mmol} / \mathrm{L})$ & $>1,0$ & $>1,3$ & $<1,0$ & $<1,3$ & $<0,9$ & $<1,0$ \\
\hline $\begin{array}{l}\text { Glucemia } \\
\text { en ayunas } \\
(\mathrm{mmol} / \mathrm{L})\end{array}$ & \multicolumn{2}{|c|}{$<5,6$} & \multicolumn{2}{|c|}{$\geq 5,6$} & \multicolumn{2}{|c|}{$\geq 6,1$} \\
\hline $\begin{array}{l}\text { Triglicéridos } \\
(\mathrm{mmol} / \mathrm{L})\end{array}$ & \multicolumn{2}{|c|}{$<1,5$} & \multicolumn{2}{|c|}{$\geq 1,5$} & \multicolumn{2}{|c|}{$\geq 1,7$} \\
\hline $\begin{array}{l}\text { Presión arterial } \\
(\mathrm{mmHg})\end{array}$ & \multicolumn{2}{|c|}{$<130 / 85$} & \multicolumn{2}{|c|}{$\geq 130 / 85$} & \multicolumn{2}{|c|}{$\geq 140 / 90$} \\
\hline
\end{tabular}

$\mathrm{H}$ : hombre; M: mujer; PC: perímetro de la cintura; HDL-C: lipoproteína de alta densidad. Caso positivo de síndrome metabólico (SM): $\geq$ de 3 alteraciones de los componentes según criterios número 1 de la puntuación ( $\mathrm{PC}, \mathrm{HDL}-\mathrm{C}$, glucemia y $\mathrm{PA}$ ) y concentración de triglicéridos $\geq 1,7 \mathrm{mmol} / \mathrm{L}$ y/o evaluación $\leq 4$ puntos de dos de los componentes del SM. Caso negativo: $\leq$ de 1 alteración de los componentes y/o evaluación $\leq$ de 2 puntos de dos de los componentes del SM.

\section{TÉCNICAS E INSTRUMENTOS DE RECOLECCIÓN DE DATOS}

Se aplicó un cuestionario para la recolección de datos como la edad, sexo, medicamentos empleados, y antecedentes clínicos como la PA. El médico investigador obtuvo los valores bioquímicos a partir de las historias clínicas.

\section{EVALUACIÓN DE LAS VARIABLES ANTROPOMÉTRICAS}

A los sujetos se les midió el peso, talla, perímetros de cintura $(P C)$ y de cadera; se calculó el IMC. La medida del PC se tomó con una cinta métrica con el sujeto colocado de pie, en espiración, con el abdomen relajado, tomando como referencia el punto medio entre el borde inferior de la última costilla y la espina ilíaca anterosuperior de cada lado. En los casos de abdómenes péndulos la medición se realizó en el punto más prominente del abdomen. El perímetro de cadera se tomó como la circunferencia máxima trazada sobre los trocánteres femorales. Se calculó el ICC con los datos obtenidos.

\section{PRESIÓN ARTERIAL}

Se realizó la medición de la PA a cada sujeto con esfigmomanómetro con mango de acorde al tamaño del brazo. Previamente, el sujeto estuvo sentado en reposo durante los diez minutos. El procedimiento se realizó tres veces en el brazo derecho del sujeto, con un intervalo de cinco minutos. El valor final de la PA correspondió al promedio de las tres mediciones obtenidas. 


\section{PROCEDIMIENTO DE LAS VARIABLES BIOQUIMÍCAS}

La obtención de la muestra sanguínea (basal) de cada sujeto, tomada luego de 8-12 horas de ayuno, sirvió para las determinaciones bioquímicas. Se midieron las concentraciones plasmáticas de glucosa, insulina y lípidos (triglicéridos y HDL-C). A todos los sujetos se les determinó glucemia e insulinemia en dos ocasiones separadas (basal y a los cinco minutos). Para el cálculo del índice de RI se tomaron los valores promediados de glucemia e insulinemia.

\section{EVALUACIÓN DE LAS VARIABLES DE LABORATORIO}

La concentración de glucosa en ayunas, triglicéridos y HDL-C se midieron en analizador automático (Elimat, Francia) por métodos enzimáticos. La concentración de insulina en muestra de sangre venosa (plasma) se determinó por un método inmunoradiométrico (IRMA, Izotop, Hungría). El índice de RI fue calculado por el modelo homeostático de Mathews (RI-HOMA): insulina en ayunas $(\mu \mathrm{Ul} / \mathrm{mL})$ x glucosa en ayunas (mmol/L) / 22, $5^{(22,23)}$. Se consideró RI cuando los valores del índice RI-HOMA fueron $\geq 2,6^{(23)}$.

Se determinó la presencia de SM en todos los individuos de acuerdo a los criterios antes descritos; también se evaluó la relación de estas definiciones con la RI. La definición de la JIS fue considerada como el estándar de oro para el diagnóstico del SM.

\section{ANÁLISIS DE DATOS}

Para los cálculos estadísticos se utilizó el programa SPSS versión 11.5 para Windows. Se calcularon medidas de resumen de acuerdo al tipo de variables: cualitativas (frecuencias y porcentajes) y cuantitativas (media y desviación estándar). Se usó la prueba homogeneidad para establecer diferencias estadísticamente significativas entre proporciones.

Se calculó la concordancia entre las distintas definiciones de SM a través del coeficiente kappa de Cohen. La interpretación del coeficiente kappa se realizó en base a los seis niveles de fuerza de concordancia propuestos por Landis y Koch (24): $\leq 0,00$ (pobre); 0,01-0,20 (leve); 0,21-0,40 (aceptable), 0,41-0,60 (moderada); 0,61-0,80 (considerable); 0,81-1,00 (casi perfecta). Se realizó el cálculo de la sensibilidad, especificidad, valores predictivos positivos (VPP) y negativos (VPN) para identificar la presencia de SM y de la capacidad de estas definiciones para detectar sujetos con RI.

En todos los casos se trabajó para un nivel de confianza del 95\% (IC 95\%), un error alfa de 0,05. Se admitieron como valores de significación estadística los valores de $p$ menores a 0,05 .

\section{ASPECTOS ÉTICOS}

El estudio fue aprobado por el Comité de Ética e Investigación del Instituto Nacional de Endocrinología (Facultad de Medicina de La Habana). Todos los participantes firmaron el consentimiento informado escrito de acuerdo a la Declaración de Helsinki, previa información de los objetivos y propósitos del estudio. Se mantuvo la confidencialidad de los datos.

\section{RESULTADOS}

Se estudiaron 350 sujetos con sobrepeso y obesidad entre los 19 y 70 años; la edad media fue de $41,6 \pm 11,25$ años; el sexo más frecuente fue el femenino (82,9\%). El 15,4\% tenía sobrepeso, un $84,6 \%$ obesidad y el $57,7 \%$ (202/350) poseían altos valores de PA o tratamiento antihipertensivo. Las características de la muestra se describen en la tabla 2.

La Tabla 3 muestra la frecuencia de SM en los sujetos con sobrepeso y obesidad según las definiciones de la OMS, EGIR, NCEP-ATPIII, IDF, AHA/NHBLI, JIS y según Szabo. Se observó que la mayor frecuencia de casos con SM fue detectada por los criterios de Szabo $(74,3 \%)$, proporción que tuvo diferencias significativas al compararlas con el resto de las definiciones empleadas en el estudio. La definición que tuvo menor frecuencia correspondió a la dada por la OMS $(42,0 \%)$.

La frecuencia de RI en los sujetos estudiados fue del $53,7 \%$. Al comparar la frecuencia de RI en los sujetos con SM utilizando las definiciones que no llevan a la RI como prerrequisito: NCEP-ATPIII $(64,3 \%, 126 / 196)$, IDF $(64,4 \%, 132 / 205)$, AHA/NHBLI $(65,9 \%, 122 / 185)$, JIS $(64,4 \%, 132 / 205)$ y según Szabo $(61,2 \%, 159 / 260)$; se encontró que la RI se distribuyó de manera similar con

Tabla 2. Características generales de los sujetos con sobrepeso y obesos $(n=350)$

\begin{tabular}{lrc}
\hline Variable & Media & $\begin{array}{c}\text { Desviación } \\
\text { estándar }\end{array}$ \\
\hline Edad (años) & 41,6 & 11,3 \\
Peso $(\mathrm{kg})$ & 94,4 & 19,3 \\
Talla $(\mathrm{cm})$ & 162,7 & 8,3 \\
IMC $\left(\mathrm{kg} / \mathrm{m}^{2}\right)$ & 35,6 & 6,3 \\
PA sistólica $(\mathrm{mmHg})$ & 123,9 & 19,8 \\
PA diastólica $(\mathrm{mmHg})$ & 83,1 & 14,0 \\
Glicemia en ayunas $(\mathrm{mmol} / \mathrm{L})$ & 4,9 & 0,9 \\
Insulinemia en ayunas $(\mu \mathrm{UI} / \mathrm{mL})$ & 15,8 & 11,2 \\
RI-HOMA & 3,5 & 2,7 \\
Triglicéridos $(\mathrm{mmol} / \mathrm{L})$ & 1,6 & 0,8 \\
HDL-C (mmol/L) & 1,2 & 0,3 \\
\hline
\end{tabular}

IMC: índice de masa corporal; PA: presión arterial; RI-HOMA: índice de resistencia a insulina según modelo homeostático de Mathews; HDL-C: Lipoproteína de alta densidad. 
Tabla 3. Frecuencia, sensibilidad, especificidad y valores predictivos de los criterios de SM para identificar sujetos con resistencia a la insulina $(n=350)$

\begin{tabular}{|c|c|c|c|c|c|}
\hline \multirow{2}{*}{ Definición de SM } & \multirow{2}{*}{$\frac{S M}{\mathrm{n}(\%)}$} & \multicolumn{4}{|c|}{ Resistencia a la insulina (\%) } \\
\hline & & S (IC 95\%) & E (IC 95\%) & VPP & VPP \\
\hline OMS* $^{*}$ & $147(42,0)$ & $91,2(86,2-96,1)$ & $73,4(67,1-79,7)$ & 71,3 & 91,9 \\
\hline$E G I R^{*}$ & $164(46,8)$ & $83,5(77,6-89,5)$ & $72,6(65,9-79,3)$ & 72,9 & 83,4 \\
\hline NCEP-ATP III & $196(56,0)$ & $64,3(57,3-71,3)$ & $59,7(51,7-67,8)$ & 67,0 & 56,8 \\
\hline IDF & $205(58,6)$ & $64,4(57,6-71,2)$ & $61,4(53,1-69,7)$ & 70,2 & 54,9 \\
\hline AHA/NHLBI & $185(52,9)$ & $65,9(58,9-73,0)$ & $60,0(52,2-67,8)$ & 64,9 & 61,1 \\
\hline JIS & $205(58,6)$ & $64,4(57,6-71,2)$ & $61,4(53,1-69,7)$ & 70,2 & 54,9 \\
\hline Según Szabo & $260(74,3) \dagger$ & $61,2(55,0-67,3)$ & $67,8(57,6-78,0)$ & 84,6 & 37,7 \\
\hline
\end{tabular}

* Definiciones que incluyen como prerrequisito la resistencia a la insulina (RI). † vs. OMS, EGIR, NCEP-ATPIII, AHA/NHLBI, IDF, JIS (p<0,0001). Total de sujetos con RI: 188. SM: síndrome metabólico; S: sensibilidad; IC 95\%: intervalo de confianza al 95\%; E: especificidad; VPP: valor predictivo positivo; VPN: valor predictivo negativo; OMS: Organización Mundial de la Salud; EGIR: European Group for the Study of Insulin Resistance; NCEP-ATPIII: National Cholesterol Education Program - Adult Treatment Panel III; AHA/NHLBI: American Heart Association / National Heart, Lung and Blood Institute; IDF: International Diabetes Federation; JIS: Joint Interim Statement (declaración conjunta de la IDF, AHA, Federación Mundial del Corazón, Sociedad Internacional de Ateroesclerosis, Asociación Internacional para el Estudio de la Obesidad y la NHLBI).

una frecuencia superior al $60 \%$ en todos los criterios. En los sujetos sin SM la frecuencia de RI se mostró de la siguiente forma: NCEP-ATPIII 40,3\% (62/154), IDF $38,6 \%$ (56/145), AHA/NHBIL 40,0\% (66/165), JIS 38,6\% (56/145) y según Szabo 32,2\% (29/90) (Tabla 3). La RI fue mucho más frecuente en los sujetos con SM para cada una de las definiciones en relación a los sujetos $\sin \operatorname{SM}(p<0,0001)$.

También se halló la sensibilidad, especificidad, VPP y VPN de los diferentes criterios de SM para identificar sujetos con RI-HOMA $\geq 2,6$. Se comprobó que los criterios que no incluían a la RI como prerrequisito (NCEP-ATPIII, IDF, AHA/NHBLI, JIS y Szabo) tenían una sensibilidad superior a $60 \%$ y VPP superiores al $64 \%$ para detectar sujetos con RI. Dentro de estos destaca Szabo con un VPP de $84,6 \%$ para el diagnóstico de RI. Tanto las definiciones de la OMS como de la EGIR son las únicas útiles para detectar el SRI en personas no diabéticas, pues incluyen a la RI como prerrequisito. ${ }^{(2,5,7)}$.
Las definiciones que tenían más alta sensibilidad $(100 \%)$ y VPN (100\%) en relación con la guía JIS fueron la NCEP-ATPIII, IDF, AHA/NHBLI, mientras que los criterios que tenían más alta especificidad (100\%) y VPP (100\%) fueron la IDF y Szabo (Tabla 4).

El análisis de concordancia diagnóstica entre las definiciones para SM se muestra en la tabla 5. La concordancia entre los criterios de Szabo y la $\mathrm{AHA}$ NHLBI fue de 0,559 (moderada), mientras que el coeficiente kappa entre los criterios de Szabo, y el resto de las guías (NCEP-ATPIII, IDF, JIS) fue considerable de 0,612 a 0,657 , respectivamente.

La guía JIS mostró un grado de acuerdo casi perfecto con las guías de la IDF, NCEP-ATPIII y AHA/NHLBI $(1,000,0,947$ y 0,885 , respectivamente), y un grado de acuerdo considerable con los criterios de Szabo $(0,657)$. Similarmente, los resultados de la IDF concordaron casi perfectamente con las guías de la AHA/NHLBI y

Tabla 4. Precisión de diagnóstico de los distintos criterios del SM en los adultos con sobrepeso y obesidad

\begin{tabular}{rrrrrr}
\hline \multicolumn{1}{l}{ Definición de SM } & & S (IC 95\%) (\%) & E (IC 95\%) (\%) & VPP (\%) & VPN (\%) \\
\hline & OMS & $83,7(77,7-89,9)$ & $59,6(52,6-62,6)$ & 60,0 & 83,4 \\
\multirow{3}{*}{ JIS } & EGIR & $81,7(75,5-87,9)$ & $61,8(54,6-69,1)$ & 65,4 & 79,3 \\
& NCEP-ATP III & $100(99,7-100)$ & $94,2(90,1-98,2)$ & 95,6 & 100 \\
& IDF & $100(99,8-100)$ & $100(99,7-100)$ & 100 & 100 \\
& AHA/NHLBI & $100(99,7-100)$ & $87,9(82,6-93,2)$ & 90,3 & 100 \\
\hline
\end{tabular}

SM: síndrome metabólico; S: sensibilidad; IC 95\%: intervalo de confianza al 95\%; E: especificidad; VPP: valor predictivo positivo; VPN: valor predictivo negativo; OMS: Organización Mundial de la Salud; EGIR: European Group for the Study of Insulin Resistance; NCEP-ATPIII: National Cholesterol Education Program - Adult Treatment Panel III; AHA/NHLBI: American Heart Association / National Heart, Lung and Blood Institute; IDF: International Diabetes Federation; JIS: Joint Interim Statement (declaración conjunta de la IDF, AHA, Federación Mundial del Corazón, Sociedad Internacional de Ateroesclerosis, Asociación Internacional para el Estudio de la Obesidad y la NHLBI). 
Tabla 5. Análisis de concordancia diagnóstica por coeficiente kappa entre los distintos criterios de SM

\begin{tabular}{|c|c|c|c|c|}
\hline \multicolumn{2}{|c|}{ Definición de SM } & \multirow{2}{*}{$\begin{array}{c}\text { Kappa } \\
0,358\end{array}$} & \multirow{2}{*}{$\begin{array}{c}\begin{array}{c}\text { Error } \\
\text { estándar }\end{array} \\
0,038\end{array}$} & \multirow{2}{*}{$\begin{array}{c}\begin{array}{c}\text { Grado de } \\
\text { concordancia }\end{array} \\
\text { Aceptable }\end{array}$} \\
\hline \multirow{6}{*}{$\begin{array}{l}\text { Según } \\
\text { Szabo }\end{array}$} & OMS & & & \\
\hline & EGIR & 0,401 & 0,041 & Aceptable \\
\hline & NCEP-ATPIII & 0,612 & 0,040 & Considerable \\
\hline & IDF & 0,657 & 0,040 & Considerable \\
\hline & AHA/NHLBI & 0,559 & 0,040 & Moderado \\
\hline & JIS & 0,657 & 0,040 & Considerable \\
\hline \multirow{5}{*}{ OMS } & EGIR & 0,602 & 0,043 & Moderado \\
\hline & NCEP-ATPIII & 0,400 & 0,047 & Aceptable \\
\hline & IDF & 0,410 & 0,045 & Moderado \\
\hline & AHA/NHLBI & 0,422 & 0,047 & Moderado \\
\hline & JIS & 0,410 & 0,045 & Moderado \\
\hline \multirow{4}{*}{ EGIR } & NCEP-ATPIII & 0,410 & 0,048 & Moderado \\
\hline & IDF & 0,429 & 0,047 & Moderado \\
\hline & AHA/NHLBI & 0,402 & 0,049 & Aceptable \\
\hline & JIS & 0,429 & 0,047 & Moderado \\
\hline \multirow{3}{*}{ NCEP-ATP III } & IDF & 0,947 & 0,017 & Casi perfecta \\
\hline & AHA/NHLBI & 0,914 & 0,022 & Casi perfecta \\
\hline & JIS & 0,947 & 0,017 & Casi perfecta \\
\hline \multirow{2}{*}{ IDF } & AHA/NHLBI & 0,885 & 0,025 & Casi perfecta \\
\hline & JIS & 1,000 & $<0,001$ & Casi perfecta \\
\hline AHANHLBI & JIS & 0,885 & 0,025 & Casi perfecta \\
\hline
\end{tabular}

SM: síndrome metabólico; OMS: Organización Mundial de la Salud; EGIR: European Group for the Study of Insulin Resistance; NCEPATPIII: National Cholesterol Education Program - Adult Treatment Panel III; AHA/NHLBI: American Heart Association / National Heart, Lung and Blood Institute; IDF: International Diabetes Federation; JIS: Joint Interim Statement (declaración conjunta de la IDF, AHA, Federación Mundial del Corazón, Sociedad Internacional de Ateroesclerosis, Asociación Internacional para el Estudio de la Obesidad y la NHLBI). En todos los casos $p<0,0001$.

del NCEP-ATPIII. El grado de acuerdo fue también casi perfecto entre los criterios de la AHA/NHLBI y del NCEPATPIII. Finalmente, los resultados de la OMS mostraron un grado de concordancia aceptable y moderada entre 0,358 y 0,422 con los criterios de Szabo, NCEPATPIII, IDF, AHA/NHLBI y JIS, pero con la EGIR una concordancia de 0,602 (Tabla 5).

\section{DISCUSIÓN}

Existe un consenso en el campo médico de que el término de SM es aceptable por la condición de la presencia de múltiples factores de riesgo de ECV y DM2 (1-3,10,17,25-27); sin embargo, existen diferencias en las frecuencias de SM al aplicar distintas definiciones.

En nuestro estudio, se aplicaron siete métodos para clasificar el SM en un grupo de sujetos con sobrepeso y obesidad. Las frecuencias encontradas de SM fueron superiores al aplicar los criterios de Szabo (74,3\%), seguidas de JIS e IDF (58,6\% en ambos casos), NCEPATPIII $(56,9 \%)$ y AHA/NHLB $(52,9 \%)$. Las definiciones de la OMS y la EGIR detectaron menor proporción de sujetos con SM $(42,0 \%$ y $46,8 \%$, respectivamente).
En investigaciones que abordan el SM en población adulta en Latinoamérica, la frecuencia osciló entre 14 al $27 \%$ (NCEP-ATPIII) ${ }^{25)}$; otros estudios latinoamericanos mostraron una frecuencia entre el 17,9 al 48,5\% (IDF y NCEP-ATPIII) ${ }^{(14,20,26)}$. En Cuba, en la provincia de Sancti Spíritus, el SM prevaleció en un 39,8\% (según criterio ALAD) ${ }^{(15)}$ y en Europa fluctuó entre 21,5 al 69,4\% (NCEP. ATPIII e IDF) ${ }^{(2,3)}$. La prevalencia del SM es mucho más dramática cuando incrementa el IMC ${ }^{(1,2,15)}$. En Kuwait, la frecuencia del SM en obesos fue del 76,9\% (IDF) ${ }^{(27)}$ y en Cuba en una cohorte de adultos donde el $78 \%$ de ellos presentaban sobrepeso y obesidad, la presencia del SM (NCEP-ATPIII) fue del $58,2 \%{ }^{(28)}$ y del $69,5 \%$ en sujetos obesos ${ }^{(16)}$.

Pocos estudios comparan simultáneamente más de cuatro definiciones del SM ${ }^{(18,20,21,29)}$. En otras poblaciones de Latinoamérica, Europa y Asia se han encontrado prevalencias similares de SM, hallándose concordancia entre las guías aplicadas ${ }^{(1,18,20,21,25,29)}$. Tales estudios han considerado válidas las definiciones de la IDF, NCEP. ATPIII, AHA/NHLBI y la JIS.

La frecuencia del SM en el resto de las definiciones utilizadas en nuestro estudio (OMS, EGIR, NCEP-ATPIII, IDF, AHA/NHLBI y JIS), oscilan entre 42,0 y $58,6 \%$; las cuales son semejantes a las halladas en otros estudios europeos (Inglaterra, Finlandia, Suecia, Holanda, Polonia, España e Italia) ${ }^{(2,3,18,30)}$ y a lo reportado en Cuba para población adulta con sobrepeso y obesidad $(16,28)$. En cambio, la frecuencia de SM según Szabo en los sujetos con sobrepeso y obesos de nuestro estudio, es superior a lo reportado en Cuba para la población adulta en general y para los sujetos con sobrepeso y obesos por la definición NCEP-ATPIII $(15,16,28)$.

La diferencia considerable encontrada en la frecuencia de SM al usar los criterios de Szabo con el resto de las definiciones estaría dada por el aporte añadido de los distintos valores de punto de corte utilizados, de cada uno de los componentes del SM (Tabla 1) y porque también se incluyen sujetos con solo dos componentes del SM; que también repercutiría en el incremento de la detección de sujetos con SM.

En esta investigación la frecuencia de RI en los sujetos con sobrepeso y obesos fue del $53,7 \%$. Un dato interesante de esta investigación, es que no existió diferencia significativa en cuanto a la presencia de $\mathrm{RI}$ para cada una de las definiciones estudiadas que no llevara implícita la RI como prerrequisito (NCEPATPIII, IDF, AHA/NHBLI, JIS y según Szabo). Por otro lado, también corroboramos que la RI estuvo asociada entre los sujetos con SM comparados con los que no lo presentaban ${ }^{(2,3,19)}$. En realidad ninguna de las definiciones analizadas logró discriminar un mayor número de SM con RI. Kocelak ${ }^{(18)}$ encontró resultados semejantes a los nuestros en la que ninguna de las 
definiciones que utilizó (IDF y NCEP-ATPIII) reveló más sujetos de SM con RI.

En cuanto a la capacidad predictiva de los distintos criterios de SM con respecto a la RI cuantificada por HOMA, señalaremos que todos los criterios de SM, que no tenían como prerrequisito la RI, mostraron valores aceptables similares de sensibilidad (entre el 61,2 al $65,9 \%$ ), especificidad (entre el 59,7 al $67,8 \%$ ) y VPP (entre el 64,9 al 84,6\%) para identificar sujetos con RI; en cambio los criterios de Szabo mostraron un mayor VPP $(84,6 \%)$ y VPN $(37,2 \%)$ (Tabla 3$)$; estos resultados nos indican que este criterio presenta una mayor precisión en la detección de sujetos con RI en comparación con el resto de las definiciones, pero al mismo tiempo exhibe una mayor proporción de individuos sin RI que tienen SM. Otros estudios han encontrado que las definiciones de NCEP-ATP III, IDF y la JIS mostraron una sensibilidad entre el 45,7 al $54,3 \%$, especificidad entre $65 \%$ al $89,6 \%$ y VPP (entre el 33,3 al 62,7\%) para detectar sujetos con $\mathrm{RI}^{(30,31)}$; resultados que son cercanos a los nuestros para estas guías (sensibilidad: 64,3 y $64,4 \%$, especificidad: 59,7 y $61,4 \%$ y VPP: 67,0 y 70,2 , respectivamente). En síntesis, ninguno de los criterios empleados (salvo OMS y EGIR) mostró una alta sensibilidad y especificidad para identificar sujetos con RI.

En relación a comparar la definición de JIS con los demás criterios estudiados, encontramos que la JIS identifica a todos los sujetos diagnosticados por las guías de la NCEP-ATPIII, IDF y AHA/NHLBI (100\% de sensibilidad). Sin embargo, los criterios de Szabo e IDF no detectan falsos positivos ( $100 \%$ especificidad) y poseen una alta probabilidad para detectar realmente los sujetos diagnosticados con SM según la JIS (VPP: 100\%) (Tabla 4). En este estudio la definición de la IDF en relación con la JIS mostró una sensibilidad y especificidad del $100 \%$. Esta coincidencia se debe a que todos los individuos sobrepeso y obesos diagnosticados por ambas guías presentaron el criterio de obesidad abdominal. En población general donde existen personas con peso adecuado, sobrepeso y obesos probablemente el diagnóstico no coincidiría, porque la IDF presenta la obesidad abdominal como criterio necesario y la JIS es flexible, dígase, incluye otros criterios y no necesariamente la obesidad abdominal como requisito obligatorio.

Nuestros datos confirman los hallazgos obtenidos por otros investigadores en relación a que las definiciones del NCEP-ATPIII, IDF, AHA/NHLBI y la JIS sean consideradas efectivas para vaticinar el SM en las poblaciones debido a que son las que muestran una mayor sensibilidad $(18,20,21,29,32)$. No obstante, los criterios de Szabo podrían ser provechosos para el diagnóstico del SM por su alta especificidad y VPP.

Esta investigación reveló un grado de concordancia desde moderado a casi perfecto según el coeficiente kappa entre las frecuencias de SM obtenidas empleando los criterios de Szabo y las guías NCEP-ATPIII, IDF, AHA/NHBLI y JIS, y no así entre estas y las definiciones de la OMS y la EGIR, en cuyo caso los niveles de concordancia fueron aceptable y moderado (Tabla 5). Este resultado coincide con lo observado por varios autores en otros países, donde se han encontrado grados de similitud sustanciales entre las guías más recientes (NCEP-ATPIII, IDF, AHA/NHBLI y JIS) ${ }^{(20,21,29,32)}$, con ligeras diferencias que pueden ser explicadas por las discrepancias en los puntos de corte de los criterios que componen el SM. Las concordancias mayores en general fueron entre las definiciones NCEP-ATPIII, IDF, AHA/NHBLI y la JIS.

La limitación principal de este estudio radica en que el muestreo fue no probabilístico por lo que los resultados no pueden ser extrapolables a la población general. En Cuba, este es el primer estudio en el cual se comparan distintas definiciones del SM en sujetos con sobrepeso $y$ obesos.

En la actualidad existen diferencias en el diagnóstico del SM al emplear las distintas definiciones en adultos. Aunque el grado de concordancia según el coeficiente kappa no sugiere la idoneidad de alguna guía en particular, las frecuencias obtenidas entre las guías analizadas en este estudio (JIS, IDF, NCEP-ATPIII, AHA/NHBLI y los criterios de Szabo) podrían sugerir su aplicación como las más adecuadas.

Los resultados del presente estudio concluyen que los criterios de Szabo revelaron una concordancia considerable con las guías NCEP-ATPIII, IDF y JIS, además de una similar frecuencia de sujetos con RI en relación al resto de las definiciones. El SM según los criterios de Szabo pudiera ser otra alternativa para la pesquisa activa del SM en poblaciones.

En resumen, el SM es muy común en los sujetos con sobrepeso y obesidad (frecuencia entre el 42,0 y el $74,3 \%$ ). El grado de desacuerdo entre las diferentes definiciones del SM indica la necesidad de una definición que sea universalmente aceptada, con una buena capacidad predictiva de la RI. Se necesitan más estudios para dar respuesta a este escenario clínico.

Fuentes de financiamiento: autofinanciado.

Conflictos de interés: los autores declaran no tener conflictos de interés.

Contribución de autoría: ECR, BS, JR, ICD, AA y NO realizaron la concepción y diseño del artículo, la recolección de datos y redacción de artículo. ECR, ICD, WC y AA realizaron el análisis e interpretación de los datos. JR y RE realizaron la asesoría técnica y administrativa. Todos los autores aprobaron la versión final del artículo. 


\section{REFERENCIAS BIBLIOGRÁFICAS}

1. Kassi E, Pervanidou P, Kaltsas G, Chrousos G. Metabolic syndrome: definitions and controversies. BMC Med. 2011;9:48. doi:10.1186/17417015-9-48

2. Samson SL, Garber AJ. Metabolic syndrome. Endocrinol Metab Clin Nprth Am. 2014;43(1):1-23. doi: 10.1016/j.ecl.2013.09.009

3. Lam DW, LeRoith D. Metabolic Syndrome. En: De Groot LJ, Chrousos G, Dungan K, et al., editores. Endotext [Internet]. South Darmouth: MDtext. com; 2015 [citado 20 dic 2016]. Disponible en: https://www.ncbi.nlm. nih.gov/books/NBK278936

4. Reaven G. Insulin resistance and coronary heart disease in nondiabetic individuals. Arterioscler Thromb Vasc Biol. 2012;32(8):1754-9. doi: 10.1161/ATVBAHA.111.241885

5. Alberti KG, Zimmet PZ. Definition, diagnosis and classification of diabetes mellitus and its complications. Part 1: diagnosis and classification of diabetes mellitus provisional report of a WHO consultation. Diabet Med. 1998;15(7):539-53.

6. Expert Panel on Detection Evaluation, and Treatment of High Blood Cholesterol in Adults. Executive Summary of the Third Report of the National Cholesterol Education Program (NCEP) Expert Panel on Detection, Evaluation, and Treatment of High Blood Cholesterol in Adults (Adult Treatment Panel III). JAMA. 2001;285(19):2486-97.

7. Balkau B, Charles MA. Comment on the provisional report from the WHO consultation. European Group for the Study of Insulin Resistance (EGIR). Diabet Med. 1999;16(5):442-3.

8. Grundy SM, Brewer HB Jr, Cleeman JI, Smith SC Jr, Lenfant C: Definition of metabolic syndrome: report of the National Heart, Lung, and Blood Institute/American Heart Association conference on scientific issues related to definition. Arterioscler Thromb Vasc Biol. 2004;24(2):e13-8. 10.1161/01. ATV.0000111245.75752.C6

9. Alberti KG, Zimmet PZ, Shaw J. The metabolic syndrome - a new worldwide definition. Lancet. 2005;366(9491):1059-62. doi: $10.1016 / \mathrm{S} 0140-6736(05) 67402-8$
10. Alberti KG, Eckel RH, Grundy SM, Zimmet PZ, Cleeman JI, Donato $\mathrm{KA}$, et al. Harmonizing the metabolic syndrome: a joint interim statement of the International Diabetes Federation Task Force on Epidemiology and Prevention; National Heart, Lung, and Blood Institute; American Heart Association; World Heart Federation; International Atherosclerosis Society; and International Association for the Study of Obesity. Circulation. 2009;120(16):1640-5. doi: 10.1161/ CIRCULATIONAHA.109.192644

11. Szabo de Edelenyi F, Goumidi L, Bertrais S, Phillips C, Macmanus $\mathrm{R}$, Roche $\mathrm{H}$, et al. Prediction of the metabolic syndrome status based on dietary and genetic parameters using Random Forest. Genes Nutr. 2008;3(34):173-6. doi: 10.1007/s12263-0080097-y

12. Song YM, Sung J, Lee K. Genetic and environmental relationships of metabolic and weight phenotypes to metabolic syndrome and diabetes: the healthy twin study. Metab Syndr Relat Disord. 2015;13(1):36-44. doi: $10.1089 /$ met.2014.0087

13. Ramphal L, Zhang J, Suzuki S. Ethnic disparities in the prevalence of the metabolic syndrome in American adults: data from the Examination of National Health and Nutrition Examination Survey 1999-2010. Proc (Bayl Univ Med Cent). 2014;27(2):92-5.

14. Wong-McClure RA, Gregg EW, Barceló A, Lee K, Abarca-Gómez L, Sanabria-López L, et al. Prevalence of metabolic syndrome in Central America: a cross-sectional populationbased study. Rev Panam Salud Publica. 2015;38(3):202-8.

15. Bustillo E, Pérez Y, Brito A, González A, Montero D, Santos M, et al. Síndrome metabólico un problema de salud no diagnosticado. Rev Cubana Endocrinol. 2011;22(3):167-81.

16. Hernández $M$, Miguel $P$, Marrero $M$, Rodríguez T, Niño S. Caracterización del síndrome metabólico en pacientes adultos con obesidad. MEDISAN. 2012;16(3):341-48.

17. Salazar MR, Carbajal HA, Espeche WG, Dulbecco CA, Aizpurúa M, Marillet AG, et al. Relationships among insulin resistance, obesity, diagnosis of the metabolic syndrome and cardio-metabolic risk. Diab Vasc Dis Res. 2011;8(2):109-16. doi: $10.1177 / 1479164111403170$

18. Kocelak P, Chudek J, OlszaneckaGlinianowicz M. Prevalence of metabolic syndrome and insulin resistance in overweight and obese women according to the different diagnostic criteria. Minerva Endocrinol. 2012;37(3):247-54.

19. Karnchanasorn R, Ou HY, Chuang LM, Chiu KC. Insulin resistance is not necessarily an essential element of metabolic syndrome. Endocrine. 2013;43(1):92-9. doi: 10.1007/s12020-012-9702-3

20. Mora G, Salguedo G, Ruíz M, Ramos E, Alario A, Fortich A, et al. Concordancia entre cinco definiciones de síndrome metabólico. Cartagena, Colombia. Rev Esp Salud Pública. 2012;86(3):301-11.

21. Wen J, Yang J, Shi Y, Liang Y, Wang F, Duan X, et al. Comparisons of different metabolic syndrome definitions and associations with coronary heart disease, stroke, and peripheral arterial disease in a rural Chinese population. PLoS One. 2015;10(5):e0126832. doi:10.1371/journal.pone.0126832

22. Mathews DR, Hosker JP, Rudenski AS, Nailor BA, Treacher DF, Turner RC. Homeostasis model assessment: insulin resistance and beta-cell function from fasting plasma glucose and insulin concentration in man. Diabetologia. 1985;28(7):412-9.

23. Arranz C, González RM, Álvarez A, Rodríguez B, Reyes A. Criterios de referencia para los indicadores de secreción de insulina y de los parámetros lipídicos en una población mixta hospitalaria. Rev Cubana Endocrinol. 2010;21(1):1-12.

24. Landis JR, Koch GG. The measurement of observer agreement for categorical data. Biometrics. 1977;33(1):159-74.

25. Escobedo J, Schargrodsky H, Champagne B, Silva H, Boissonnet $\mathrm{C}$, Vinueza $\mathrm{R}$, et al. Prevalence of the metabolic syndrome in Latin America and its association with subclinical carotid atherosclerosis: the CARMELA cross sectional study. Cardiovascular Diabetol. 2009;8:52. doi: $10.1186 / 1475-2840-8-52$ 
26. Rosas J, González A, Aschner P, Bastarrachea R, et al. Epidemiología, diagnóstico, control, prevención y tratamiento del síndrome metabólico en adultos. Rev ALAD. 2010;18(1):2544.

27. Al Zenki S, Al Omirah H, Al Hooti S, Al Hamad N, Jackson RT, Rao A, et al. High prevalence of metabolic syndrome among Kuwaiti Adults - A wake-up call for public health intervention. Int J Environ Res Public Health. 2012;9(5):1984-96. doi: 10.3390/ijerph9051984

28. Rodriguez-Ojea A, Alonso C, Yarnell JW, Woodside JV. Status of novel cardiovascular risk factor and cardiovascular disease risk in an urban Cuban population - A Pilot Study. J Health Popul Nutr. 2011;29(5):510-5.
29. Khosravi-Boroujeni H, Ahmed F, Sadeghi M, Roohafza H, Talaei M, Dianatkhah M, et al. Does the impact of metabolic syndrome on cardiovascular events vary using different definitions? BMC Public Health. 2015;15:1313. doi: 10.1186/s12889-015-2623-3

30. Martínez J, Franch J, Romero J, Cánovas C, Gallardo A, López ML. Capacidad predictiva de los criterios diagnósticos del síndrome metabólico sobre la resistencia a la insulina y el riesgo coronario. Med Clin (Barc). 2007;129(16):601-6.

31. Hosseinpanah F, Borzooei S, Barzin M, Farshadi M, Sarvghadi F, Azizi F. Diagnostic values of metabolic syndrome definitions for detection of insulin resistance: Tehran Lipid and Glucose Study (TLGS). Arch
Iran Med. 2012;15(10):606-10. doi: 0121510/AIM.006

32. Esmailzadehha N, Ziaee A, Kazemifar AM, Ghorbani A, Oveisi S. Prevalence of metabolic syndrome in Qazvin Metabolic Diseases Study (QMDS), Iran: a comparative analysis of six definitions. Endocr Regul. 2013;47(3):111-20.

Correspondencia: Dr. Eduardo Cabrera Rode, PhD.

Dirección: Instituto Nacional de Endocrinologia, Zapata y D, $10400 . \mathrm{La}$ Habana, Cuba.

Teléfono: (+537) 8326298

Correo electrónico: eduardo.cabrerarode@ gmail.com

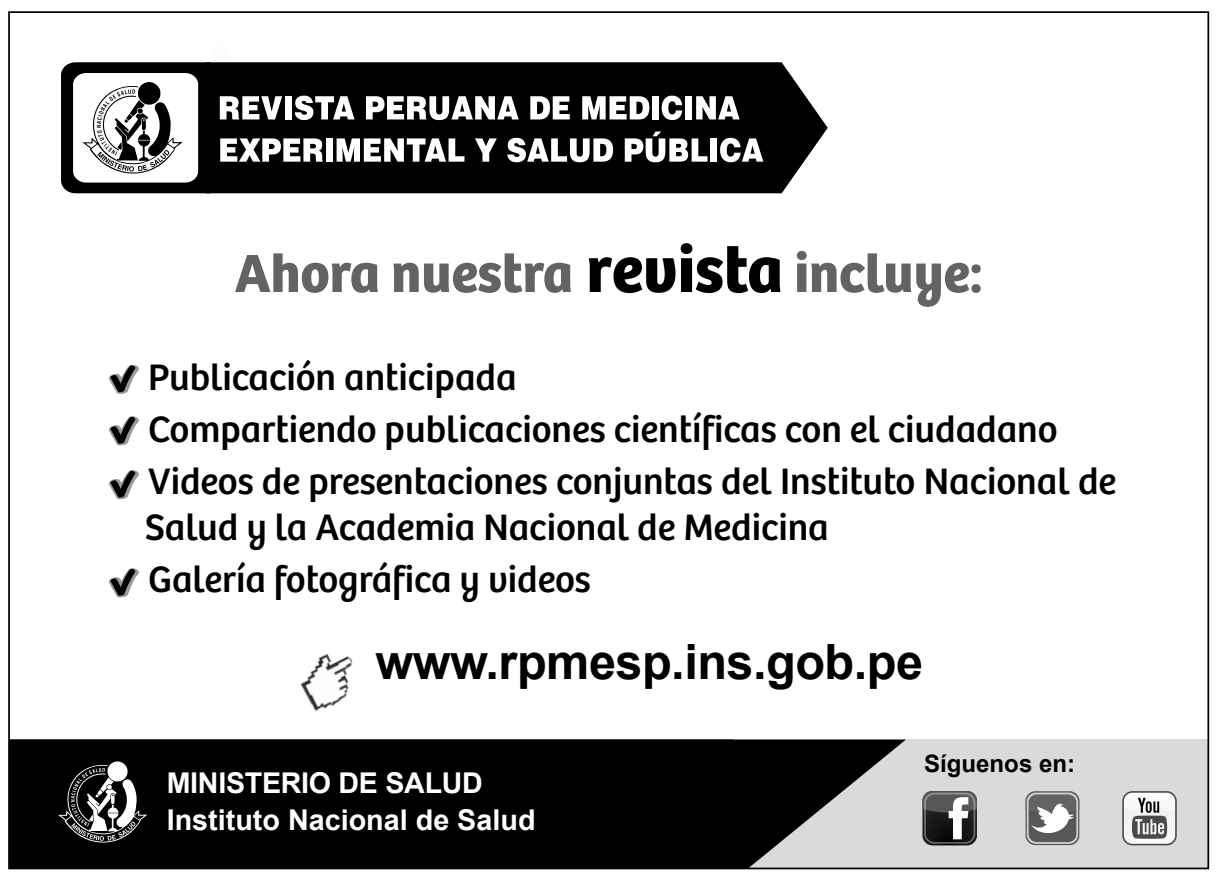

